\title{
Polyploidization and epigenetics
}

\author{
LI ZhiHui ${ }^{1}$, LU Xiang ${ }^{1}$, GAO Yun ${ }^{2}$, LIU ShaoJun ${ }^{3}$, TAO Min ${ }^{3}$, XIAO Heng ${ }^{1}$, QIAO YunQian ${ }^{1}$, \\ ZHANG YaPing ${ }^{2,4}$ \& LUO Jing ${ }^{1,4^{*}}$ \\ ${ }^{1}$ School of Life Sciences, Yunnan University, Kunming 650200, China; \\ ${ }^{2}$ State Key Laboratory of Genetic Resources and Evolution, Kunming Institute of Zoology, Chinese Academy of Sciences, Kunming 650223, \\ China; \\ ${ }^{3}$ School of Life Sciences, Hunan Normal University, Changsha 410081, China; \\ ${ }^{4}$ Laboratory for Conservation and Utilization of Bio-resource \& Key Laboratory for Microbial Resources of the Ministry of Education, Yunnan \\ University, Kunming 650091, China
}

Received September 3, 2010; accepted October 25, 2010

\begin{abstract}
The occurrence of polyploidy, or whole genome duplication, can result in instantaneous speciation. Because new polyploids are immediately reproductively isolated from their non-polyploid congeners, polyploidization has played an important role in the diversification of flowering plants and some vertebrates. Newly formed polyploids must respond to this instantaneous genomic change, which resembles "genome shock" syndrome to survive and reproduce successfully. Epigenetic changes, which do not cause changes to the sequence of DNA, can significantly contribute to the survival of and ultimately to the evolutionary success of new polyploids. Epigenetic regulation, both transcriptional and post-transcriptional, entails changes in DNA methylation, gene status and/or nucleolus dominance. These changes provide effective and flexible ways for a new polyploid to respond quickly to the enormous change in genetic material, to survive and potentially reproduce. We examine and assess certain epigenetic phenomena and possible pathways that may facilitate the evolutionary success of polyploid organisms.
\end{abstract}

polyploidization, epigenetics, DNA methylation, gene status, nucleolus dominance

Citation: $\quad$ Li Z H, Lu X, Gao Y, et al. Polyploidization and epigenetics. Chinese Sci Bull, 2011, 56: 245-252, doi: 10.1007/s11434-010-4290-1

\section{Occurrence of polyploidy and its evolutionary significance}

Polyploidy is an important evolutionary feature of flowering plants and vertebrates [1,2]. Most angiosperms and many animals have undergone this process many times [3]. There are indications that most vertebrates are paleopolyploids, derived from an ancestor in which two rounds of genome doubling or whole genome duplication (WGD) occurred. Teleosts, which are the most species-rich group of vertebrates, are derived from a lineage that experienced a third round of WGD [4-8]. Study of the production, survival and subsequent success of polyploids may provide insight into

*Corresponding author (email: jl.jingluo@gmail.com) some of the more dramatic evolutionary events, and epigenetic changes may be crucial to this understanding.

\section{Polyploidization and phenotypic expression}

Two types of polyploids can be recognized, allopolyploids and autopolyploids. Allopolyploids are often derived from a two-step process: hybridization between two species and then doubling of all the chromosomes by the union of unreduced gametes (or doubling may occur by other mechanisms) [9]. In contrast, autopolyploids are usually formed by chromosome doubling within a species [9].

Contemporary work on polyploidization mainly focuses on the duplicated genome and evolution post-polyploidization, e.g., the occurrence of polysomic inheritance after genome 
doubling, changes in genome structure, gene structure, gene expression, and subsequent phenotypic changes. Current studies suggest that once polyploidy occurs, whatever the mechanism involved, the polyploid genome evolves rapidly [10].

Epigenetic changes promote the survival and reproduction of neopolyploids and subsequently facilitate genomic changes. Subsequent to polyploidization, genomic shock syndrome and dramatic changes are likely to produce severe stress on the survival and reproduction of polyploids. Chen [10] argued that "Epigenetic changes, which are potentially reversible, provide an effective and flexible means for a polyploid cell to respond to polyploidy or genomic shock. Moreover, gene silencing or activation that is initially epigenetic and reversible could be one step toward a genetically fixed and irreversible state". Observed phenomena provide clues to the potential effects of polyploidizationdependent epigenetic changes. For example, the single genome of an autopolyploid is the same as its parents' except in the number of chromosomes, yet the phenotype of an autopolyploid may be distinguishably different from that of its parents. Compared to chromosome doubling, inter-specific hybridization exerts greater effects on gene expression [9]. Gene expression changes affect the phenotypes of organisms. For example, allopolyploids (or interspecific hybrids) can present various phenotypes, some of which may be inherited from their parents, some of which are newly formed and some of which are lost in polyploid offspring. In newly formed Tragopogon allopolyploids, transcriptional investigation indicated that $5 \%$ of genes have been silenced and that $4 \%$ exhibited novel patterns of expression [11]. These results suggest that such expression changes may be epigenetically controlled.

\section{Epigenetics versus genetics}

Epigenetics generally refers to heritable changes in gene expression that do not involve DNA mutations or chromosome changes (genetics) [12 and therein]. Epigenetic regulation of gene expression occurs by DNA and/or histone modification. In polyploids, epigenetically produced phenomena include gene silencing, gene activation, genomic imprinting, maternal effects, nucleolus dominance and chromatin conformation changes.

\section{Phenotypic variation and hybrid vigor in polyploids}

Many studies have demonstrated that genetic and epigenetic changes, triggered by hybridization and whole genome duplication, play major roles in phenotypic expression [13-16], which in turn determines the fitness of newly formed polyploids [17]. Several important crops such as wheat, cotton and canola are allopolyploid and contain two or more different genomes [10,18]. Some animals, including several commercially raised fish are polyploid, such as Carassius auratus gibelio [19], allotetraploid progenies of Carassius auratus crossed with Cyprinus carpio and allotriploids produced by crossing allotetraploid progenies of Carassius auratus $\times$ Cyprinus carpio with C. auratus cuvieri [20,21]. These crops and fish breeds show more phenotypic variation and hybrid vigor than their parents or progenitors [18-21]. For example, segregating hybrids and stable allopolyploids of Arabidopsis thaliana and Arabidopsis arenosa are larger than their parents [18]. In vertebrates, the growth rates of triploid C. a. gibelio, allotriploids produced by crossing allotetraploid Carassius auratus $\times$ Cyprinus carpio progeny with $C$. auratus cuvieri and allotetraploid progeny of Carassius auratus $\times$ Cyprinus carpio were $13.7 \%-34.4 \%$ [19], about $100 \%$ [20] and $21.8 \%$ [21] faster than their parents, respectively. The latest evidence indicates that newly formed genomic interactions in allopolyploids induce phenotypic variation and increased growth [18]. Furthermore, the over-dominance model suggests that heterosis should reach its peak at the maximum levels of heterozygosity and dissipate when approaching homozygosity [22].

Once successful polyploidization has occurred, genome complexity and fitness can be enhanced to produce greater genomic flexibility. This can result in higher growth rates, larger size and increased survival fitness in harsher environments.

\section{Epigenetic phenomena and relevant mechanisms in polyploidy}

\subsection{Polyploidization and DNA methylation}

(i) DNA methylation in polyploids. The production of polyploids is often accompanied by alterations in DNA methylation. Some studies $[16,23]$ found that changes in DNA methylation patterns occurred more frequently in synthetic allotetraploids than in their parents. Cytosine methylation is a universal epigenetic phenomenon [24,25], and is the most important epigenetic change associated with plant polyploidy [26-28]. For example, tobacco possesses a highly repeat-rich allotetraploid genome in which about $28 \%$ of cytosines appeares to be methylated [29]. In addition, DNA methylation changes are observed in newly formed allopolyploids of Arabidopsis [16] and wheat [30]. Many A. thaliana genes were transcriptionally suppressed in resynthesized allotetraploids. In natural allopolyploids, a subset of A. thaliana loci, including transposons and centromeric repeats, were heavily methylated and subjected to homologous genome-specific RNA-mediated DNA methylation; however, reduced methylation at some loci was also observed [22]. In a synthetic allotetraploid (Aegilops sharonensis $\times$ Ae. umbellulata), some genetic alterations were 
associated with comparative hypomethylation of the promoter region within the Ae. umbellulata-derived rDNA units [31]. In another example, the endoreduplication of cells during tomato fruit ripening was not accompanied by material changes in global methylation levels; there was no change in the level of methylation, but the patterns of methylation had changed [32]. This suggests that methylation patterns may be rapidly established on dramatically multiplied chromosomes [23].

(ii) Mechanisms of DNA methylation. What causes alternations of methylation in a polyploid? Recent findings have indicated alternative possibilities. Firstly, methyltransferases are responsible for methylation, and disruption of met 1 expression affects gene regulation and development in allopolyploids [22]. Chen et al. [16,22] treated resynthesized and natural Arabidopsis allotetraploids with 5-aza-2'deoxycytosine (5-aza-dC), a chemical inhibitor of DNA methyltransferases, and observed dramatic changes in phenotypes. Protein-coding genes [22,33] were also reactivated, suggesting that DNA methylation was responsible for reactivation of some genes in the allotetraploids [22]. Hence, the expression level of methyltransferases plays a crucial role in gene expression, gene regulation, growth and development. Methyltransferase 1 (MET1) mainly affects CG methylation in allotetraploids, and an increase in DNA methylation is likely to be caused by an indirect effect of a met 1 defect [22]. The increase in DNA methylation in the promoter region is probably associated with the down-regulation of this gene in a genetic background that is predominantly demethylated. This is reminiscent of the dense methylation that is observed around the transcription start site and within the coding region of the SUPERMAN (SUP) gene in $A$. thaliana, which over-expresses antisense MET1 [22,34]. In allopolyploids, down-regulation of MET1 led to an overall reduction of $\mathrm{CG}$ methylation and to increased levels of transcription of the SCP repetitive gene family and of En/Spm transposons and centromeric repeats, suggesting a causal link between CG methylation maintained by MET1 and transposon suppression [10]. Secondly, Chen [10] suggested that allopolyploidy and DNA methylation might have distinct and overlapping effects on gene regulation in allopolyploid genomes. They also observed that both the density and the specific sites of DNA methylation could affect gene regulation [10]. Reduced DNA methylation can cause severe growth and developmental abnormalities, and the severity increases in self-fertilized progeny. This is probably because hypomethylation induces other changes in the genome [22], as observed in the selfed progeny of ddm1 mutants $[35,36]$. Thirdly, changes in DNA methylation patterns occur more frequently in synthetic allotetraploids than in their parents [16,23]. This is perhaps related to the silencing of "redundant" genes in a doubled genome, or maybe because DNA methylation systems are perturbed by the effects of interspecies hybridization and polyploidization [23]. Fulnecek et al. [23] speculated that in tobacco, which has a repeat-rich heterochromatic genome, natural selection might favor additive expression of parental DNA methyltransferase genes that maintain high levels of DNA methylation. Methylation of repeat sequences could also be important for their stability in the cell nucleus [37], decreasing their recombination frequency [38] and preventing intergenomic homogenization [39]. Recent studies suggest that DNA methylation is likely to be involved in harmonizing genome structure and expression after genome merging in allopolyploid plants [23]. Therefore, maintaining high levels of DNA methylation may be important for an organism. Polyploidization can induce changes of DNA methylation, and DNA methylation plays a pivotal role in genome regulation and gene expression, thereby affecting growth, development and phenotype. Subsequent to polyploidization, organisms possess novel characteristics, different from those of their parents.

(iii) Levels of DNA methylation related to gene status. There may be close relationships between levels of DNA methylation and gene status [40,41]. The studies of Chen et al. [22] suggested that allopolyploidization induced DNA methylation changes in genes that were suppressed in synthetic allopolyploids, and reduced DNA methylation induced activation of suppressed genes in natural allopolyploids. Both polyploidization (interspecific hybridization) and DNA methylation affect the status of certain genes in A. thaliana. These genes are, however, generally suppressed in natural allotetraploids [10] and are sensitive to changes in DNA methylation [22]. Suppression or activation of these genes in a polyploid is likely to result in different levels of expression. Chen et al. [22] studied the relationship between gene status (gene silencing or gene activation), and the pathways involved. First, for homologous-specific centromeric DNA methylation, methylation of some homologous loci (e.g., locus At2g23810) was correlated with gene suppression in resynthesized and natural Arabidopsis allotetraploids [22]. Second, to test if loss of DNA methylation was associated with gene activation in allopolyploids of Arabidopsis, Chen et al. [22] analyzed DNA methylation variations in reactivated genes and transposons using DNA blot analysis. Their results indicated that activation of At5g36180 (SCP), At4g08010 and At1g44070 was related to reduced CG methylation [22]. Third, At2g23810 encoded a putative senescence-associated protein (SAP1). SAP1 was expressed at high levels in A. thaliana and was suppressed in resynthesized (F5) allotetraploids [22,42], while SAP1 was silenced in the natural allotetraploid A. suecica. Silencing of this gene correlated with hypermethylation of both $\mathrm{CG}$ and non-CG sites; however, when the overall methylation levels of both $\mathrm{CG}$ and non-CG sites were dramatically reduced, SAP1 was reactivated [22]. Fourth, when DNA methylation was reduced in the natural allotetraploid A. suecica, the expression of about 200 genes was altered [22]. These results indicate that DNA methylation or hypermethylation can result in gene suppression or silencing, and reduced DNA 
methylation or hypomethylation can lead to gene activation. Other investigations with different organisms, using alternative methods support the above conclusion [22,33,43-46].

\subsection{Polyploidization and gene silencing}

(i) Gene silencing in polyploids. Numerous cases of gene silencing have been reported in polyploids. For example, diploid $A$. thaliana line $\mathrm{C}$ outcrosses to tetraploid plants can result in reduced hygromycin phosphotransferase (HPT) transgene activity [47]. Silencing of orthologous genes from one progenitor was estimated to occur at a frequency of $\sim 0.4 \%$ in synthetic allotetraploids and $\sim 2.5 \%$ in natural allotetraploids, with genes of $A$. thaliana and A. arenosa being equally likely to be silenced [43]. Sometimes, parallel expression and silencing patterns are observed in natural and neopolyploids that are of similar genomic constitution $[44,48,49]$; wheat allohexaploids also show organ-specific silencing of homologs [9]. Adams et al. [48] documented considerable variation in the expression and silencing patterns of homologous genes in different organs of allopolyploid Gossypium hirsutum. Indeed, unequal expression of homologs and silencing of one copy have been observed in neopolyploids, as shown by several studies of allotetraploid Gossypium, Triticum and Arabidopsis [13,45,49-51]. These studies indicate that gene silencing is widespread following direct or indirect polyploidization.

Subfunctionalization may result in genes that are reciprocally silenced in polyploids. Adams et al. [48,52] showed reciprocal, organ-specific silencing of homologs of the alcohol dehydrogenase gene $A d h A$ in various floral organs of $G$. hirsutum, such that one homolog had been silenced in some organs and the other homolog had been silenced in other organs. This was then followed by subfunctionalization [9]. Subfunctionalization may lead to changes in gene expression levels and gene expression patterns, ultimately resulting in phenotypic differentiation [2,53]. Moreover, in allotetraploids, after the formation of epialleles, genes will be reciprocally silenced [45]. In summary, reciprocal silencing of genes may be a new epigenetic event, and it affects gene expression levels and thus potentially may change phenotypes.

(ii) Silencing of redundant genes due to balance dosage. The underlying molecular mechanism of gene silencing may result from the requirement to shut down redundant gene copies. Pikaard [43] noted that when chromosomes pair, there would be some 'chatter' in new allopolyploids, and that "evidence emerged that new allopolyploids might deal with these challenges by silencing some of the redundant 'chatter' and by finding ways to reduce the incidence of chromosomal infidelity", meaning that changes of ploidy seem to result in epigenetic silencing [47]. In addition, he noted that "transposable elements, the troublesome hounds of the genome, are often unleashed in new hybrids and can roam their new environment causing damage, which ultimately leads to gene silencing" [43]. Simultaneously, to reduce damage, cells may also limit changes in gene expression by silencing some gene regions [43].

\subsection{Polyploidization and nucleolus dominance}

(i) Nucleolus dominance in polyploids. Nucleolus dominance in hybrids and allopolyploids of both plants and animals mainly describes rRNA gene silencing [54], where rRNA genes are highly methylated [55-57] and silenced over one or several loci $[58,59]$. Nucleolus dominance is a widespread epigenetic phenomenon [58,59], which is exhibited by diploids and allopolyploids $[31,59,60]$. Nucleolus dominance has been observed in both plants and animals, including Drosophila interspecific hybrids and Xenopus, Arabidopsis, Brassica and wheat allopolyploids [61,62]. Nucleolar dominance does not, however, appear to be part of a broader genome silencing phenomenon [62]. Dadejova et al. [39] reported the establishment of epigenetic patterns of rRNA gene expression in synthetic hybrids of Nicotiana species, and Pikaard [43] found that synthetic allotetraploids that recreated four naturally occurring Brassica or Arabidopsis allotetraploid species displayed the same patterns of nucleolar dominance observed in natural allotetraploids, beginning as early as the $\mathrm{F}_{1}$ generation and becoming completely established by $\mathrm{F}_{2}$.

(ii) Possible mechanisms of altering nucleolus dominance. Nucleolus dominance is reversible and developmentally regulated and is controlled by chromatin modifications involving DNA methylation and histone acetylation $[10,58,63]$. Blocking histone acetylation or DNA methylation suppresses the silenced rRNA genes [10]. In Arabidopsis and Brassica, the nucleolar dominance phenomenon was found to be related to allopolyploid formation and subsequent DNA methylation and histone modifications [46,58,63,64]. The role of chromatin modifications in silencing or activating protein-coding genes in allopolyploids has been reported in several recent studies [16,33,44,45,65,66]. Silenced rRNA genes subjected to nucleolar dominance are suppressed by 5 -aza-dC (a chemical inhibitor of cytosine methylation) or by chemical inhibitors of histone deacetylation, indicating a role for chromatin modifications in the silencing process $[58,64]$.

As early as 1982, Martini et al. [67] suggested that the dominant effect of U-genome nucleolus organizer regions (NORs) was determined by the large number of repeats in the IGS (intergenic spacer) compared with those in other Triticum species. Reeder [62] proposed the hypothesis of an "enhancer imbalance mechanism" to explain nucleolar dominance, in which there was competition between IGS repeats for enhancer elements in limiting supply. Shcherban et al. [31] suspected that the repeats in the insertion served as a transcription enhancer that promoted higher activity of the Ae. umbellulata-derived NORs in different hybrid combinations. Another appealing hypothesis to explain nucleolar dominance is that dominant rRNA genes have the 
most transcriptional enhancers $[62,63,68]$. In plants, the evidence supporting this hypothesis, however, is indirect and still under survey [63].

Chen and Pikaard [63] suggested that reactivation of suppressed rRNA genes in B. napus was associated with the developmental transition from the inflorescence to the floral meristem. Likewise, Wilson et al. [69] showed that nucleolar dominance was complete in early embryos of Xenopus hybrids, but transcripts from under-dominant genes could be detected late in embryonic development and in organs of adult frogs. Therefore, in both animals and plants there is evidence that nucleolar dominance is developmentally regulated independent of gamete formation.

Although nucleolar dominance phenomena are often found in polyploids, there seems to be no relationship between nucleolar dominance and polyploidization. For instance, in $B$. carinata, whose tetraploid genome is derived from B. oleracea and B. nigra, 6 clones had $100 \%$ identity to the promoter of B. oleracea and 4 clones had $100 \%$ identity to the $B$. nigra promoter [63]. No sequence polymorphisms were observed in the promoter region sequenced [63]. These data suggest that rRNA gene promoter sequences are unchanged since the formation of the polyploid, presumably thousands of years ago [63] and are in agreement with the view of Prakash and Hinata [70]. Thus, nucleolar dominance is independent of ploidy [63]. Furthermore, Chen and Pikaard [63] pointed out that nucleolar dominance was also independent of maternal effect, rRNA gene dosage, size of repetitive region or number of repetitive elements.

The mechanisms for the establishment and maintenance of nucleolar dominance are still poorly understood [31,71]; there is no single hypothesis that can explain the range of expression patterns found in different organisms [31,72]. Chen and Pikaard [63] also indicated that we could not explain nucleolar dominance according to expression levels of rRNA genes in plants. Other work indicates that under-dominant rRNA genes are subjected to suppression, suggesting that enhancer dosage and transcription factor availability are also unlikely to explain all aspects of nucleolar dominance [63]. Chen and Pikaard [63] stated that experiments were needed to determine whether changes in DNA methylation were a cause or an effect of nucleolar dominance and to explain how dominant and underdominant genes were first discriminated within the nucleus. Future tasks will be to determine whether nucleolar dominance is a hybrid-specific dosage compensation mechanism or a product of the same mechanisms controlling the number of active rRNA genes during normal development [63].

\section{Other epigenetic events and causes of epigenetic alterations}

Ongoing studies indicate that RNA interference (RNAi) and microRNAs (miRNAs) may interfere with epigenetics in newly formed polyploids. Ha et al. [73] argued that because interspecific hybrids and allopolyploids are mergers of two sets of microRNA sequences and divergent genomes, accumulation of miRNA and target sites might be influenced. RNAi affects gene status via gene silencing and gene suppression, and this feature has been used to study epigenetics $[18,22]$.

miRNAs are associated with non-additive expression of target genes in allotetraploids. Genome merging in allotetraploids induces genetic and epigenetic changes [10], leading to non-additive expression of miRNA targets and miRNA primary transcripts [73]. Bartel [74] proposed that non-additive accumulation of miRNAs might be caused by non-additive expression of miRNA biogenesis genes. At the post-transcriptional level, non-additive expression of miRNA biogenesis genes resulted in non-additive accumulation of miRNAs [75]. This non-additivity is partly associated with transcriptional regulation of miRNA loci and their targets $[10,73,75]$. Many microRNA (miRNA) targets are nonadditively expressed in the allotetraploids [42,73], suggesting a role for miRNAs in buffering genetic clashes between species $[73,75]$.

Ha et al. [73] studied the natural allotetraploid Arabidopsis, and found that miRNA sequences were conserved between species, but that their expression patterns were highly variable between the allotetraploids and their progenitors. There can be rapid and dramatic changes of miRNA expression levels in allopolyploids, for the following reasons: miRNAs play an important role in maintaining flower morphology and development in stable allotetraploids; miRNAs mediate expression diversity between closely related species and in allotetraploids [73]; many miRNA targets encode transcription factors or proteins that are important for growth and development in plants and animals [73,74]; non-additive regulation of some miRNAs and their targets may lead to novel phenotypes in allopolyploids [73]. Interestingly, many miRNAs accumulate differently between diploid Arabidopsis and its allotetraploid (A. suecica), indicating a role for miRNAs in allopolyploid evolution. Expression variation of miRNAs can lead to changes of gene expression, growth vigor, and adaptation [73].

To validate the effects of RNAi on gene status, Chen et al. [22] carried out RNAi on methyltransferase 1 (metl) using the allotetraploid A. suecica. RNAi of met1 reduced DNA methylation and altered the expression of some genes, many of which encoded transposons, predicted proteins, and centromeric and heterochromatic RNAs. Some transposons were also activated and even gene At2g23810 was demethylated [22]. When At2g23810 and genes that encode transposons were activated, gene expression levels were subsequently changed. Developmental pathways of $A$. suecica must be quite different from those of its parents $A$. thaliana and $A$. arenosa.

Kovarik et al. [76] suggested that there might be a potential link between rDNA homogenization and epigenetics 
based on a study of rDNA evolution in Nicotiana allopolyploids. Following allopolyploidy, rDNA may homogenize, leading to reduced complexity and a decreased number of rDNA variants $[31,77,78]$. Rapid evolution and/or amplification of a new rDNA family and its homogenization between rDNA loci have previously been observed in a synthetic tobacco allopolyploid [79]. Although the causes of DNA sequence homogenization need to be determined, it undoubtedly influences growth and development of organisms.

\section{Conclusions and perspectives}

After polyploidization, one epigenetic event or mechanism is not independent of another; phenotypes of organisms are determined by converging effects (Figure 1). DNA methylation is widespread in polyploids and can lead to gene silencing. Nucleolar dominance is an epigenetic phenomenon that describes rRNA gene silencing, which is controlled by chromatin modifications involving DNA methylation and histone acetylation. Furthermore, the role of chromatin modifications in silencing or activating protein-coding genes in allopolyploids is important. Therefore, investigations into epigenetic events and relevant polyploidization pathways are in demand.

Epigenetic phenomena are involved in the early events of neopolyploid formation and provide flexibility for polyploid progeny to respond to the substantial changes of a doubled genome. The study of how epigenetic phenomena facilitate polyploidization will elucidate vital events in evolutionary history and the early stages of speciation, and of how polyploidization can lead "to an increase in biological complexity and the origin of evolutionary novelties" [2].

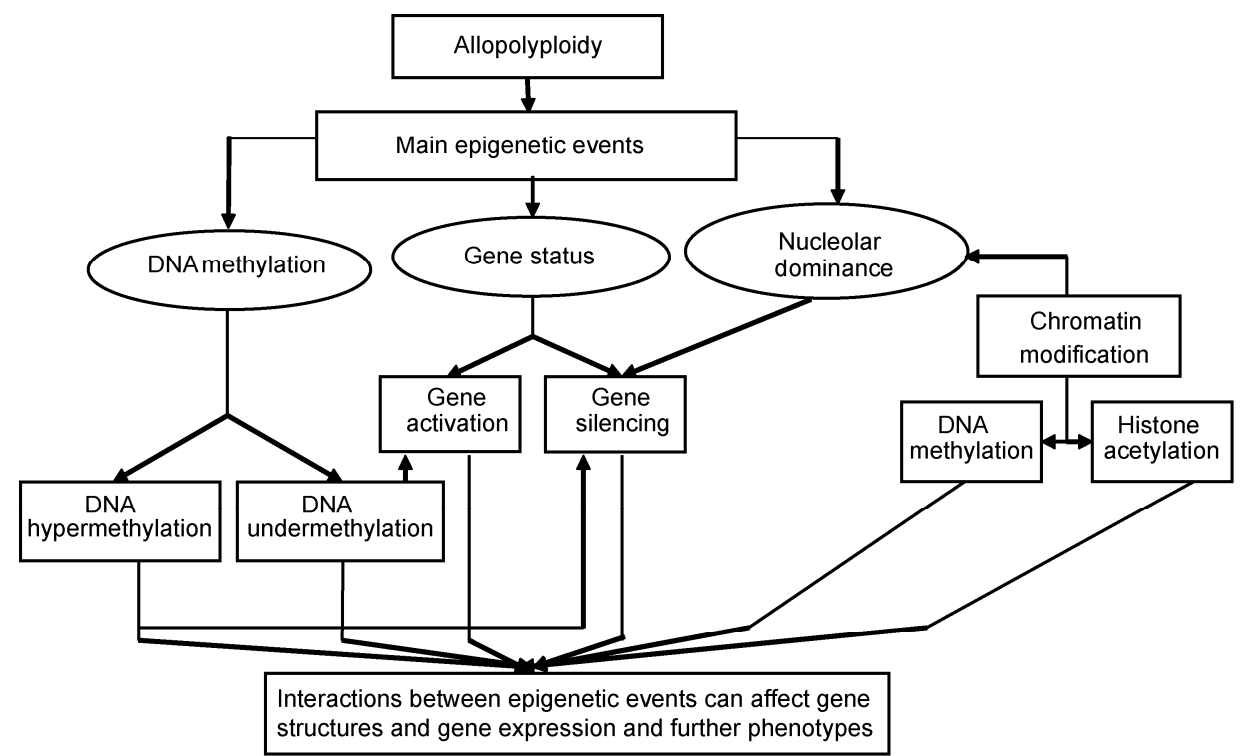

Figure 1 Main epigenetic events and mechanisms and their potential relationships after allopolyploidization.

This work was supported by the National Basic Research Program of China (2007CB411600), the National Natural Science Foundation of China (30870291, 30930071), the National Undergraduate Students Research Training Fund of the State Education Ministry, the National Fund for Fostering Talents of Basic Science (J0730652), the Research Training Fund from the Experiment Teaching Center of Life Sciences, YNU, the Project of Higher Education Reform and Practice of Science and Engineering of the State Education Ministry (239), and the Scientific Research Foundation for Returned Overseas Chinese Scholars of the State Education Ministry. We thank S.D.L. Miller for both structure and English language revision.

1 Otto S P, Whitton J. Polyploid incidence and evolution. Annu Rev Genet, 2000, 34: 401-437

2 van de Peer Y, Maere S, Meyer A. The evolutionary significance of ancient genome duplications. Nat Rev Genet, 2009, 10: 725-732

3 Soltis D E, Soltis P S. Polyploidy: Recurrent formation and genome evolution. Trends Ecol Evol, 1999, 14: 348-352
4 Taylor J S, van de Peer Y, Braasch I, et al. Comparative genomics provides evidence for an ancient genome duplication event in fish. Philos Trans R Soc Lond B Biol Sci, 2001, 356: 1661-1679

5 Taylor J S, Braasch I, Frickey T, et al. Genome duplication, a trait shared by 22,000 species of ray-finned fish. Genome Res, 2003, 13: 382-390

6 Christoffels A, Koh E G L, Chia J, et al. Fugu genome analysis provides evidence for a whole-genome duplication early during the evolution of ray-finned fishes. Mol Biol Evol, 2004, 21: 1146-1151

7 Jaillon O, Aury J M, Brunet F, et al. Genome duplication in the teleost fish Tetraodon nigroviridis reveals the early vertebrate proto-karyotype. Nature, 2004, 431: 946-957

8 Vandepoele K, Vos W D, Taylor S J, et al. Major events in the genome evolution of vertebrates: Paranome age and size differs considerably between ray-finned fishes and land vertebrates. Proc Natl Acad Sci USA, 2004, 101: 1638-1643

9 Adams K L. Evolution of duplicate gene expression in polyploid and hybrid plants. J Hered, 2007, 98: 136-141

10 Chen Z J. Genetic and epigenetic mechanisms for gene expression and phenotypic variation in plant polyploids. Annu Rev Plant Biol, 
2007, 58: 377-406

11 Soltis D E, Soltis P S, Pires J C, et al. Recent and recurrent polyploiyd in Tragopogon (Asteraceae): Cytogenetic, genomic and genetic comparisons. Biol J Linn Soc, 2004, 82: 485-501

12 Rapp R A, Wendel J F. Epigenetics and plant evolution. New Phytol, 2005, 168: 81-89

13 Comai L, Tyagi A P, Winter K, et al. Phenotypic instability and rapid gene silencing in newly formed Arabidopsis allotetraploids. Plant Cell, 2000, 12: 1551-1568

14 Schranz M E, Osborn T C. Novel flowering time variation in the resynthesized polyploid Brassica napus. J Hered, 2000, 91: 242-246

15 Schranz M E, Osborn T C. De novo variation in life-history traits and responses to growth conditions of resynthesized polyploidy Brassica napus (Brassicaceae). Am J Bot, 2004, 91: 174-183

16 Madlung A, Masuelli R W, Watson B, et al. Remodeling of DNA methylation and phenotypic and transcriptional changes in synthetic Arabidopsis allotetraploids. Plant Physiol, 2002, 129: 733-746

17 Wendel J F, Doyle J J. Polyploidy and evolution in plants. In: Henry R J, ed. Diversity and Evolution in Plants. Oxon, UK: CAB Publishing, 2004

18 Ni Z, Kim E D, Ha M, et al. Altered circadian rhythms regulate growth vigour in hybrids and allopolyploids. Nature, 2009, 457: 327-331

19 Gui J F. New varieties of Carassius auratus gibelio for breedingBrief introduction of Zhongke No. 3 (in Chinese). Sci Fish Farm, 2009: 21

20 Zhou G J, Zhang B. Fine varieties of freshwater aquacultureCarassius auratus (in Chinese). Sci Plant Breed, 2007: 50-51

21 Liu S J, Lu Y, Zhou G J, et al. The formation of tetraploid stocks of red crucian carp $\times$ common carp hybrids as an effect of interspecific hybridization. Aquaculture, 2001, 192: 171-186

22 Chen M, Ha M, Lackey E, et al. RNAi of met1 reduces DNA methylation and induces genome-specific changes in gene expression and centromeric small RNA accumulation in Arabidopsis allopolyploids. Genetics, 2008, 178: 1845-1858

23 Fulnecek J, Matyasek R, Kovalík A. Faithful inheritance of cytosine methylation patterns in repeated sequences of the allotetraploid tobacco correlates with the expression of DNA methyltransferase gene families from both parental genomes. Mol Genet Genomics, 2009, 281: 407-420

24 Bird A. The essentials of DNA methylation. Cell, 1992, 70: 5-8

25 Richards E J, Elgin S C. Epigenetic codes for heterochromatin formation and silencing: Rounding up the usual suspects. Cell, 2002, 108: 489-500

26 Ingelbrecht I, van Houdt $\mathrm{H}$, van Montagu M, et al. Posttranscriptional silencing of reporter transgenes in tobacco correlates with DNA methylation. Proc Natl Acad Sci USA, 1994, 91: 10502-10506

27 Meyer P, Niedenhof I, Ten Lohuis M. Evidence for cytosine methylation of non-symmetrical sequences in transgenic Petunia hybrid. E J, 1994, 13: 2084-2088

28 Vanyushin B F. DNA methylation in plants. Curr Top Microbiol Immunol, 2006, 301: 67-122

29 Messeguer R, Ganal M W, SteVens J C, et al. Characterization of the level, target sites and inheritance of cytosine methylation in tomato nuclear DNA. Plant Mol Biol, 1991, 16: 753-770

30 Shaked H, Kashkush K, Ozkan H, et al. Sequence elimination and cytosine methylation are rapid and reproducible responses of the genome to wide hybridization and allopolyploidy in wheat. Plant Cell, 2001, 13: 1749-1759

31 Shcherban A B, Badaeva E D, Amosova A V, et al. Genetic and epigenetic changes of rDNA in a synthetic allotetraploid, Aegilops sharonensis $\times$ Ae. umbellulata. Genome, 2008, 51: 261-271

32 Teyssier E, Bernacchia G, Maury S, et al. Tissue dependent variations of DNA methylation and endoreduplication levels during tomato fruit development and ripening. Planta, 2008, 228: 391-399

33 Lee H S, Chen Z J. Protein-coding genes are epigenetically regulated in Arabidopsis polyploids. Proc Natl Acad Sci USA, 2001, 98: 6753-6758

34 Kishimoto N, Sakai H, Jackson J, et al. Site specificity of the Arabidop- sis METI DNA methyltransferase demonstrated through hypermethylation of the superman locus. Plant Mol Biol, 2001, 46: 171-183

35 Stokes T L, Richards E J. Induced instability of two Arabidopsis constitutive pathogen-response alleles. Proc Natl Acad Sci USA, 2002, 99: 7792-7796

36 Stokes T L, Kunkel B N, Richards E J. Epigenetic variation in Arabidopsis disease resistance. Genes Dev, 2002, 16: 171-182

37 Peng J C, Karpen G H. Epigenetic regulation of heterochromatic DNA stability. Curr Opin Genet Dev, 2008, 18: 204-211

38 Maloisel L, Rossignol J L. Suppression of crossing-over by DNA methylation in Ascobolus. Genes Dev, 1998, 12: 1381-1389

39 Dadejova M, Lim K Y, Souckova-Skalicka K, et al. Transcription activity of rRNA genes correlates with a tendency towards intergenomic homogenization in Nicotiana allotetraploids. New Phytol, 2007, 174: 658-668

40 Martienssen R, Colot V. DNA methylation and epigenetic inheritance in plants and filamentous fungi. Science, 2001, 293: 1070-1074

41 Jackson J P, Lindroth A M, Cao X, et al. Control of CpNpG DNA methylation by the KRYPTONITE histone $\mathrm{H} 3$ methyltransferase. Nature, 2002, 416: 556-560

42 Wang J, Tian L, Lee H S, et al. Genomewide nonadditive gene regulation in Arabidopsis allotetraploids. Genetics, 2006, 172: 507-517

43 Pikaard C S. Genomic change and gene silencing in polyploids. Trends Genet, 2001, 17: 675-677

44 Wang J, Tian L, Madlung A, et al. Stochastic and epigenetic changes of gene expression in Arabidopsis polyploids. Genetics, 2004, 167: 1961-1973

45 Mittelsten S O, Afsar K, Paszkowski J. Formation of stable epialleles and their paramutation-like interaction in tetraploid Arabidopsis thaliana. Nat Genet, 2003, 34: 450-454

46 Lawrence R J, Earley K, Pontes O, et al. A concerted DNA methylation/histone methylation switch regulates rRNA gene dosage control and nucleolar dominance. Mol Cell, 2004, 13: 599-609

47 Mittelsten S O, Jakovleva L, Afsar K, et al. A change of ploidy can modify epigenetic silencing. Proc Natl Acad Sci USA, 1996, 93: 7114-7119

48 Adams K L, Cronn R, Percifield R, et al. Genes duplicated by polyploidy show unequal contributions to the transcriptome and organspecific reciprocal silencing. Proc Natl Acad Sci USA, 2003, 100: 4649-4654

49 He P, Friebe B, Gill B, et al. Allopolyploidy alters gene expression in the highly stable hexaploid wheat. Plant Mol Biol, 2003, 52: 401-414

50 Kashkush K, Feldman M, Levy A A. Gene loss, silencing, and activation in a newly synthesized wheat allotetraploid. Genetics, 2002, 160: 1651-1659

51 Adams K L, Percifield R, Wendel J F. Organ-specific silencing of duplicated genes in a newly synthesized cotton allotetraploid. Genetics, 2004, 168: 2217-2226

52 Adams K L, Wendel J F. Allele-specific, bi-directional silencing of an alcohol dehydrogenase gene in different organs of interspecific diploid cotton hybrids. Genetics, 2005, 171: 2139-2142

53 Lynch M, Force A. The probability of duplicate gene preservation by subfunctionalization. Genetics, 2000, 154: 459-473

54 Pikaard C S. The epigenetics of nucleolar dominance. Trends Genet, 2000, 16: 495-500

55 Delseny $\mathrm{M}$, Laroche $\mathrm{M}$, Penon $\mathrm{P}$. Methylation pattern of radish (Raphanus sativus) nuclear ribosomal RNA genes. Plant Physiol, 1984, 76: 627-632

56 Flavell R B, O’Dell M, Thompson W F. Cysosine methylation of ribosomal RNA genes and nucleolus organizer activity in wheat. J Mol Biol, 1988, 204: 523-534

57 Torres-Ruiz R A, Hemleben V. Pattern and degree of methylation in ribosomal RNA genes in Cucurbita pepo L. Plant Mol Biol, 1994, 26 : 1167-1179

58 Chen Z J, Pikaard C S. Epigenetic silencing of RNA polymerase I transcription: A role for DNA methylation and histone modification in nucleolar dominance. Genes Dev, 1997, 11: 2124-2136

59 Pikaard C S. Nucleolar dominance: Uniparental gene silencing on a multi-megabase scale in genetic hybrids. Plant Mol Biol, 2000, 43: 
$163-177$

60 Pontes O, Lawrence R J, Neves N, et al. Natural variation in nucleolar dominance reveals the relationship between nucleolus organizer chromatin topology and rRNA gene transcription in Arabidopsis. Proc Natl Acad Sci USA, 2003, 100: 11418-11423

61 Pikaard C S. Nucleolar dominance and silencing of transcription. Trends Plant Sci, 1999, 4: 478-483

62 Reeder R H. Mechanisms of nucleolar dominance in animals and plants. J Cell Biol, 1985, 101: 2013-2016

63 Chen Z J, Pikaard C S. Transcriptional analysis of nucleolar dominance in polyploid plants: Biased expression/silencing of progenitor rRNA genes is developmentally regulated in Brassica. Proc Natl Acad Sci USA, 1997, 94: 3442-3447

64 Chen Z J, Comai L, Pikaard C S. Gene dosage and stochastic effects determine the severity and direction of uniparental rRNA gene silencing (nucleolar dominance) in Arabidopsis allopolyploids. Proc Natl Acad Sci USA, 1998, 95: 14891-14896

65 Kashkush K, Feldman M, Levy A A. Transcriptional activation of retrotransposons alters the expression of adjacent genes in wheat. Nat Genet, 2003, 33: 102-106

66 Wang J, Tian L, Lee H S, et al. Nonadditive regulation of FRI and FLC loci mediates flowering-time variation in Arabidopsis allopolyploids. Genetics, 2006, 173: 965-974

67 Martini G, O'Dell M, Flavell R B. Partial inactivation of wheat nucleolus organizers by the nucleolus organizer chromosomes from $A e$ gilops umbellulata. Chromosoma, 1982, 84: 687-700

68 Flavell R B. The structure and control of expression of ribosomal RNA genes. Oxford Surv Plant Mol Cell Biol, 1986, 3: 252-274
69 Wilson H D, Barber S C, Walter T. Loss of duplicate gene expression in tetraploid Chenopodium. Biochem Syst Ecol, 1983, 11: 7-13

70 Prakash S, Hinata K. Taxonomy, cytogenetics and origin of crop Brassicas: A review. Opera Bot, 1980, 55: 1-57

71 Pikaard C S, Lawrence R J. Uniting the paths to gene silencing. Nat Genet, 2002, 32: 340-341

72 Pikaard C S, Chen Z J. Nucleolar dominance. In: Paule M R, ed. Transcription of Ribosomal RNA Genes by Eukaryotic RNA Polymerase I. Berlin: Springer-Verlag, 1998. 277-294

73 Ha M, Lu J, Tian L, et al. Small RNAs serve as a genetic buffer against genomic shock in Arabidopsis interspecific hybrids and allopolyploids. Proc Natl Acad Sci USA, 2009, 106: 17835-17840

74 Bartel D P. MicroRNAs: Genomics, biogenesis, mechanism, and function. Cell, 2004, 116: 281-297

75 Ha M, Pang M, Agarwal V, et al. Interspecies regulation of microRNAs and their targets. Biochim Biophys Acta, 2008, 1779: 735742

76 Kovarik A, Dadejova M, Lim Y K, et al. Evolution of rDNA in Nicotiana allopolyploids: A potential link between rDNA homogenization and epigenetics. Ann Bot, 2008, 101: 815-823

77 Wendel J F. Genome evolution in polyploids. Plant Mol Biol, 2000, 42: 225-249

78 Volkov R A, Borisjuk N V, Panchuk I I, et al. Elimination and rearrangements of parental rDNA in the allotetraploid Nicotiana tabacum. Mol Biol Evol, 1999, 16: 311-320

79 Skalicka K, Lim K Y, Matyasek R, et al. Rapid evolution of parental rDNA in a synthetic tobacco allotetraploid line. Am J Bot, 2003, 90: 988-996

Open Access This article is distributed under the terms of the Creative Commons Attribution License which permits any use, distribution, and reproduction in any medium, provided the original author(s) and source are credited. 